\title{
What is new about Rio?
}

\author{
Rogerio Souza ${ }^{1}$ and Steven M. Kawut ${ }^{2}$
}

Affiliations: ${ }^{1}$ Pulmonary Dept, Heart Institute, University of São Paulo Medical School, São Paulo, Brazil. ${ }^{2}$ Dept of Medicine, Perelman School of Medicine at the University of Pennsylvania, Philadelphia, PA, USA.

Correspondence: Rogerio Souza, Pulmonary Dept, Heart Institute, University of São Paulo Medical School, São Paulo, Brazil. E-mail: souza.rogeriolame.com

@ERSpublications

Long-term use of riociguat in PAH and inoperable CTEPH provides important information on the safety of this new drug http://ow.ly/K0lxr

This issue of the European Respiratory Journal includes the results of three different studies of riociguat, a soluble guanylate cyclase stimulator, for the treatment of pulmonary hypertension [1-3]. As the first drug in a novel class for the treatment of pulmonary hypertension, riociguat has raised hopes of an expanded drug armamentarium against the still incurable diseases of pulmonary arterial hypertension (PAH) and surgically inaccessible (or persistent post-operative) chronic thromboembolic pulmonary hypertension (CTEPH). Two of these studies are long-term extension studies from pivotal phase III trials: CHEST-1 (Chronic Thromboembolic Pulmonary Hypertension Soluble Guanylate Cyclase-Stimulator Trial 1) [4] and PATENT-1 (Pulmonary Arterial Hypertension Soluble Guanylate Cyclase-Stimulator Trial 1) [5]. The third study (PATENT PLUS) studied the safety and efficacy of the combination of riociguat and the phosphodiesterase (PDE)-5 inhibitor, sildenafil.

Long-term extension studies without placebo comparator arms are useful for increasing the number of patient-days of drug exposure and providing more information about safety and tolerability. In PAH, the identification of idiosyncratic (but clinically significant) liver toxicity associated with the use of sitaxentan, a selective endothelin receptor antagonist [6], and the increased incidence of subdural haematoma reported with the use of imatinib, a tyrosine-kinase inhibitor [7], occurred in the long-term follow-up or post-marketing phase after short-term placebo-controlled randomised clinical trials suggested clinical efficacy. It is perhaps not surprising that in the realm of the relatively short placebo-controlled trials that have been conducted by sponsors (and accepted by regulatory authorities) for approval, the extension phases of trials have revealed safety issues that were not seen in the randomised portion and that ended up overshadowing the potential benefits of these two drugs.

Conversely, the evaluation of efficacy in unblinded extension studies is more challenging. The absence of an adequate randomised control group, selection bias in terms of inclusion criteria for the trial, extension study entry and retention, and use of concomitant medications for PAH (without an adequate comparative arm) make it difficult to evaluate efficacy in open-label extension studies. PATENT-2 included 396 patients from the 405 patients that completed PATENT-1, while CHEST-2 enrolled 237 patients from the 243 patients that completed CHEST-1. The high rate of transition of active subjects from the randomised trials to extension studies is commendable and $>70 \%$ of the original randomised study population remained in both studies. The results of the 6-min walk test at 1 year in the extension studies suggested a sustained effect of riociguat for improving the exercise capacity in these two forms of pulmonary hypertension, despite varying assumptions in sensitivity analyses.

Patients from the placebo group in CHEST-1 showed an improvement of $51 \mathrm{~m}$ after transition to active drug in CHEST-2, but walked about $10 \mathrm{~m}$ less than the active treatment group in CHEST-1. The sensitivity analysis for the 6-min walk test performed in PATENT-2 also showed similar results. Previous studies in $\mathrm{PAH}$ suggested that allocation to the placebo group during a randomised controlled trial was

Received: Feb 262015 | Accepted after revision: March 042015

Conflict of interest: Disclosures can be found alongside the online version of this article at erj.ersjournals.com

Copyright CERS 2015 
associated with suboptimal response to subsequent active treatment in terms of 6-min walk distance [8]; however, it is difficult to make a similar claim in the PATENT-2 or CHEST-2 studies, due to the lack of formal statistical testing and the forces of selection in the sample of patients who enter extension studies. For example, patients assigned to active treatment or placebo with clinical worsening in the randomised portion of the study may have different potentials for treatment response, such that the placebo-treated patients with the greatest potential to improve with active treatment may be the ones who do not enter the extension study. The possible differential exclusion of such "responders" makes subsequent comparisons between the groups somewhat difficult.

No major safety issues were identified during the long-term follow-up. Nonetheless, hypotension and syncope were reported side-effects. While $7 \%$ of all patients had syncope as an adverse event in both studies, hypotension was reported in $6 \%$ of patients in CHEST-2 and in 9\% of patients in PATENT-2. Taken together with the results of the trial evaluating the acute haemodynamic effects of riociguat in patients with pulmonary hypertension associated with diastolic heart failure (DILATE-1) [9], which showed $\sim 25 \%$ decrease in systemic vascular resistance with riociguat, these findings suggest that riociguat has a significant vasodilatory effect on the systemic circulation.

Vasodilation of systemic vasculature could also account for the occurrence of haemoptysis and/or pulmonary haemorrhage reported in both studies. These were reported as serious adverse events in up to $3 \%$ and as adverse events in up to $6 \%$ of all patients. The lower incidence rates of haemoptysis classified as serious adverse events in the extension studies compared with the randomised clinical trials could be attributable to the exclusion from the extension studies of those patients at highest risk. Factors such as patient age, use of anticoagulants, or severity of disease could increase the risk of this side-effect. Even considering that haemoptysis is a known complication of pulmonary hypertension [10], the occurrence of haemoptysis during treatment with riociguat warrants a prompt and comprehensive evaluation of the patient and the treatment regimen.

Riociguat stabilises NO-soluble guanylate cyclase coupling and directly stimulates soluble guanylate cyclase, increasing the production of cyclic guanosine monophosphate (cGMP) and causing vasodilation. PDE-5 inhibitors, such as sildenafil, block cGMP degradation. Therefore, the combination of both drug classes might have synergistic clinical benefit but with the potential for adverse effects on systemic blood pressure, which was the impetus for a small, double-blind, placebo-controlled randomised clinical trial evaluating the addition of riociguat to baseline treatment with sildenafil without formal hypothesis testing [1]. 12 patients were randomised to riociguat and six to placebo. There were no differences in the maximum change in supine systolic blood pressure from the baseline assessment $4 \mathrm{~h}$ after dosing of the study drug. However, patients allocated to riociguat may have had smaller increases in 6-min walk distance and greater increases in N-terminal pro-brain natriuretic peptide than patients allocated to placebo. More significantly, during the extension phase, all 17 patients using sildenafil and riociguat reported adverse events, with hypotension being the most frequent event, present in $41 \%$ of all patients. Furthermore, three deaths occurred in this small sample during a mean follow-up of 305 days. This study was terminated by the sponsor and primary investigator without a recommendation to stop by the Data Monitoring Committee. The systemic vasodilatory effects of riociguat might have been potentiated by the combination with sildenafil, resulting in a dangerous haemodynamic environment. While these findings are suggestive of an adverse effect of the combination of these two therapies, without adequate power and formal statistical testing in the randomised portion and a comparator arm in the extension, it is difficult to draw firm conclusions. Even so, this study provided important information supporting a contraindication of co-administration of riociguat and PDE-5 inhibitors in PAH treatment.

These studies have characterised the long-term use of riociguat in PAH and inoperable CTEPH, providing important information regarding the treatment profile in these conditions with this new drug from a new class. As the only drug approved by regulatory boards for CTEPH, the role for riociguat in inoperable patients is clear. The place for this treatment in the more complicated terrain of treatment for PAH may be less well defined. These studies better establish the safety profile; nevertheless, future studies and clinical experience will ultimately define the role of riociguat within the increasing number of treatment approaches for PAH.

\section{References}

1 Galiè N, Müller K, Scalise AV, et al. PATENT PLUS: a blinded, randomised and extension study of riociguat plus sildenafil in PAH. Eur Respir J 2015; 45: 1314-1322.

2 Rubin LJ, Galiè N, Grimminger F, et al. Riociguat for the treatment of pulmonary arterial hypertension: a long-term extension study (PATENT-2). Eur Respir J 2015; 45: 1303-1313.

3 Simonneau G, D'Armini AM, Ghofrani HA, et al. Riociguat for the treatment of chronic thromboembolic pulmonary hypertension: a long-term extension study (CHEST-2). Eur Respir J 2015; 45: 1293-1302.

4 Ghofrani HA, D'Armini AM, Grimminger F, et al. Riociguat for the treatment of chronic thromboembolic pulmonary hypertension. N Engl J Med 2013; 369: 319-329. 
5 Ghofrani HA, Galiè N, Grimminger F, et al. Riociguat for the treatment of pulmonary arterial hypertension. N Engl J Med 2013; 369: 330-340.

6 Galiè N, Hoeper MM, Gibbs JS, et al. Liver toxicity of sitaxentan in pulmonary arterial hypertension. Eur Respir J 2011; 37: 475-476.

7 Hoeper MM, Barst RJ, Bourge RC, et al. Imatinib mesylate as add-on therapy for pulmonary arterial hypertension: results of the randomized IMPRES study. Circulation 2013; 127: 1128-1138.

8 Rubin LJ, Badesch DB, Fleming TR, et al. Long-term treatment with sildenafil citrate in pulmonary arterial hypertension: the SUPER-2 study. Chest 2011; 140: 1274-1283.

9 Bonderman D, Pretsch I, Steringer-Mascherbauer R, et al. Acute hemodynamic effects of riociguat in patients with pulmonary hypertension associated with diastolic heart failure (DILATE-1): a randomized, double-blind, placebo-controlled, single-dose study. Chest 2014; 146: 1274-1285.

10 Tio D, Leter E, Boerrigter B, et al. Risk factors for hemoptysis in idiopathic and hereditary pulmonary arterial hypertension. PLoS One 2013; 8: e78132. 